L. Wasserman and E. Bowell

Lowell Observatory

\title{
1. THE ASTROMETRIC SYSTEM
}

The Lowell Observatory PDS is currently used in several projects which necessitate astrometric reduction of photographic plates. To meet this need, we have developed a series of computer programs for astrometric data reduction which enable the user to obtain maximum flexibility from the PDS while at the same time retaining ease of use. In its simplest form our astrometric "package" consists of four separate programs. The first two constitute the "data taking" part of the process and reside on a DEC PDP 11/34, the computer which controls the PDS. The second two programs handle "data reduction" and reside on Lowell's general purpose computer, a DEC PDP $11 / 55$.

The initial program, called PLFIND, supplies the locations on the plate of the knowns (i.e., reference stars) and the unknowns. For basic astrometric work, there are three versions of PLFIND, one for each of three available star catalogs: the SAO, AGK3 and Perth 70. Operationally, all three versions run similarly. Other options are available: for example, the user may repeat a net of reference stars previously used, a secondary reference net may be entered manually, or a set of unknowns only may be entered. When the program is invoked, the user is asked to enter a plate identifier, the plate date, the approximate plate scale, the plate center, and then the identifications of five (arbitrarily chosen) stars on the plate. The user moves the PDS platen to set on each of these five stars in turn. The program reads the manually set plate $(x, y)$ coordinates of each star, locates all five stars in the appropriate catalog (stored on disk), and does a linear plate solution based on these five fiducial stars (only an approximate plate solution is required at this stage). The user next enters a search center, search area, and maximum number of knowns desired for the fit. The search area, which need not be centered on the plate center, is the area within which known reference stars will be selected. Given the size of the search area and the linear plate solution, the program searches the star catalog, extracts all stars contained in the search area, culls this list until the desired number 
of knowns is obtained, and then computes their $(x, y)$ coordinates on the plate. At this point the user can provide positions of unknown objects. Either or both of two methods may be used. In the first, the user enters the RA and Dec of an unknown. Using the linear plate solution, the PDS moves the plate to the corresponding ( $x, y)$ coordinates. Then, depending on what is found, the user can either continue without providing an unknown, or he may adjust the PDS platen position to set exactly on the unknown. In the second method, the user moves the platen to the unknowns one by one. In either case, the user can enter an ID tag for each unknown. The output from PLFIND is a file of $(x, y)$ coordinates for both the knowns and unknowns.

This file is the input to the second program, PLSCAN, which is a stripped-down version of the Perkin-Elmer PDS driving program, SCANSALOT. PLSCAN does a raster (back-and-forth) scan centered on each $(\mathrm{x}, \mathrm{y})$ coordinate provided by PLFIND. The step size and scan length are provided by the user (a square area is always used). In most cases, a 500- $\mu \mathrm{m}$ square area is scanned, centered on each star, using a 20- $\mathrm{m}$ square aperture and a step size of $10 \mu \mathrm{m}$ so as to oversample the image. Each star scan takes about 30 seconds. This generates a $51 \times 51$ grid of density values for each star. PLSCAN writes the density grids for all the knowns and unknowns to disk. This completes the "data taking" part of the process.

At this point, the data are transferred to the 11/55 for reduction both because $11 / 55$ has fast floating point hardware and because doing the reduction on the $11 / 55$ frees the $11 / 34$ and PDS for other uses.

The first step in the reduction is to determine the exact center of each star from its density grid. This is done by program PLCTR, a heavily modified version of a program originally written by chiu (1977) to perform digital centering (separately in $x$ and $y$ ) using Gaussian fits to marginal distributions (van Altena and Auer, 1975). We have retained the fitting routines while adding more operator intervention. For example, the operator can ask to see the fit on a Tektronix 4010, the area over which the marginal distribution is taken can be changed at will, and if no fit can be obtained numerically, the operator can use the Tektronix moveable reticles as to obtain an "eyeball" fit to the the data. The output of this program is the "fitted" ( $x, y)$ coordinates. This file is the input to the final program in the chain, PLFIT, which does the plate solution and generates celestial coordinates for the unknowns. Currently, PLFIT determines a full quadratic solution, but does not take into account magnitude or color terms.

This system has been used routinely at Lowell for the past few years. It is easy to use and enables rapid plate reduction--a plate with 80 knowns and 20 unknowns can be completely reduced in about one hour. 


\section{ACCURACY AND PRECISION OF MEASUREMENTS}

As part of an occultation prediction program at Lowell, we have measured 26 plates, each containing three images of asteroid 375 Ursula and AGK $+39^{\circ} 303$. These plates, taken with the USNO Flagstaff Station 1.55-meter reflector were reduced using a secondary net of 14 stars measured by Arnold Klemola on plates obtained at the 0.5 -meter Carnegie Double Astrograph at Lick Observatory. One measure of the internal accuracy of the procedure is shown in Figure 1, where the declination measures of the star are plotted as a histogram. Of the 78 images on the plates, one of the images was not measured and a second

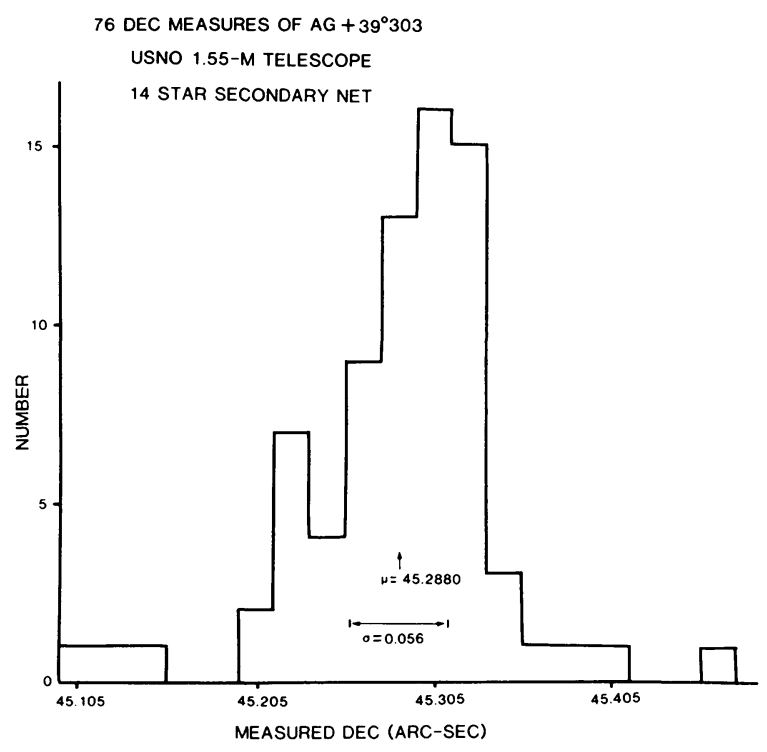

Figure 1. A histogram of 76 declination measures of an AGK star using a secondary net of 14 stars provided by A. Klemola.

was rejected since the resulting position was more than six standard deviations from the mean. As is evident from the figure, the internal accuracy is very good, particularly considering that many of the plates were taken under poor seeing conditions. A measure of the precision is given by the computed ephemeris corrections of the asteroid. These corrections measure the relative separation of the star and asteroid and are given by:

$$
\begin{aligned}
& \Delta \alpha=\left(\alpha_{a}-\alpha_{e}\right)-\left(\alpha_{*}-\alpha_{c}\right) \\
& \Delta \delta=\left(\delta_{a}-\delta_{e}\right)-\left(\delta *-\delta_{c}\right),
\end{aligned}
$$

where subscript a refers to the measured asteroid position, e refers 
to the ephemeris asteroid position, * to the measured star position, and $\mathbf{c}$ to the star catalog position. From the 26 plates we obtain $\Delta \alpha=$ $0 \$ 1004 \pm 0 \$ 0044$ and $\Delta \delta=0 ! 354 . \pm 0 ! 041$. These quantities may also be independently derived from observations of the occultation made at six sites: we have obtained $0.0992 \pm 0.0001$ and $0 ! 324 \pm 0 ! 003$, respectively (Millis et al., 1984), indicating a precision of a few hundredths of an arc second or better. This corresponds to 1 or 2 microns at the USNO plate scale of 13.55 arcseconds per $\mathrm{mm}$. Of course, part of this error is measurement error and part is due to the use of a secondary net.

Another test of the measurement accuracy, which is independent of a particular network of known stars, was carried out by the USNO Flagstaff station. They measured $(x, y)$ coordinates of nine stars on $12.7 \times 17.8 \mathrm{~cm}$ Praesepe plates taken with the 1.55-meter reflector in both a direct and "flipped" sense. The ( $x, y)$ coordinates from both sets of measures were then compared in the computer by mathematically reversing the "flipped" measures and fitting one set of measures to another by least squares. The results, for six plates, yielded a mean radial residual of $0.90 \mu \mathrm{m}$. A similar test on the USNO's SAMM machine in Washington, D. C., gave $2.27 \mu \mathrm{m}$. Also, a parallax plate was measured on the PDS. The plate yielded the same parallax as the SAMM machine, but with slightly smaller errors (Dahn, 1984).

\section{APPLICATIONS}

There are four projects which are currently using our astrometric system at Lowell or have used the system in the past. The first is a survey program of asteroid astrometry. This program, effective since mid-1979, has resulted in the measurement and publication of some 16000 asteroid and comet positions. It is thus the most prolific general survey currently in progress, and this is due largely to the ease and rapidity of the astrometric facility described here. In particular, all newly discovered asteroids have been measured in a timely way, allowing many of them to be followed up for orbit determination. It might be noted that Chiu's marginal distribution fitting algorithm works well even on highly elongated asteroid trails because centroids (in density space) are still accurately determinable.

The second project is a program to predict occultations of faint non-catalog stars by asteroids (see e,g., Wasserman et al., 1983). Plates of star fields to be traversed by selected asteroids in upcoming apparitions are searched for possible occultations. To avoid known systematic errors inherent in positions obtained with the Lowell 0.33-meter refractor, we are currently measuring plates taken by Arnold Klemola with the Carnegie Double Astrograph. A variant of the PLFIND program described above is used: we first write a file containing the asteroid's ephemeris for the interval when it will pass through the star field under analysis. Using the linear (approximate) 
plate solution derived from the five fiducial stars, the PDS platen is driven to follow this ephemeris. Thus, an operator watching the PDS screen effectively sees the greatly accelerated motion of the asteroid across the sky and can watch for stars close to the asteroid's track. When a candidate star does pass through the field, the scanning is stopped, and the PDS platen is set manually on the star. Each star selected in this way becomes an unknown whose position will subsequently be measured. Later, the ephemeris of the asteroid can be compared in detail with the measured positions of the unknown stars and occultation ground tracks can be predicted. One result of this program is shown in Figure 2. This is the ground track for the occultation of $B D+80471$ by (1) Ceres on 13 November 1984. Although the star is a BD star, it is not listed in the AGK or SAO catalogs and therefore was not identified during the course of an automated search for occultations of catalog stars. This unique event would have been missed were it not for the plate search using our astrometric system. There are no occultations of catalog stars by Ceres during this decade. If the event is sucessfully observed, it will provide an accurate density for Ceres since its mass is known.

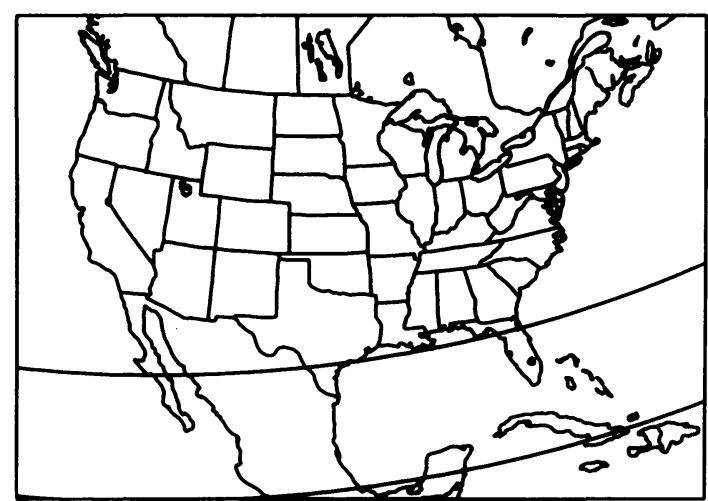

PREDICTED TRACK OF THE 13 NOVEMBER 1984 OCCULTATION OF BD+8 471 BY CERES
Figure 2. Predicted ground track of the occultation of $\mathrm{BD}+80471$ by (1) Ceres on 13 November 1984 . This event was found by a plate search technique.

A program of QSO astrometry using plates taken with the 4-meter Mayall reflector at Kitt Peak has also been carried out with this system (Hoag, Thomas and Vaucher, 1982). Here, Lowell 0.33-meter astrograph plates are used to define a secondary net of stars around the position of each QSO (the QSOs themselves are not visible on the Lowell plates). The positions of stars in the secondary net are measured using the techniques described above. Then, using versions of PLFIND and PLFIT which allow for the radial distortions, the secondary net is used to determine the position of the QSO on the 4-meter plates.

The last project has already been mentioned: refinement of occultation ground track predictions using USNO 1.55-meter plates and secondary nets provided by Klemola. This "last-minute" refinement is 
often necessary in order to locate the ground track accurately so that portable telesopes can be deployed in the field.

Note Added in Proof: The occultation by Ceres was successfully observed at twelve sites in Mexico, the Caribbean, and Florida; the actual track differed only slightly from the predicted track shown in Figure 2. A paper giving the results is in preparation.

\section{REFERENCES :}

Chiu, G.L.-T. 1977, Astron. J. 82, 842 .

Dahn, C. 1984, personal communication.

Hoag, A. A., Thomas, N. G. and Vaucher, B. G. 1982, Astrophys. J. 263,23 .

Millis, R. L., Wasserman, L. H., Bowell, E., Franz, O. G., Klemola, A., and Dunham, D. W. 1984, Astron. J. 89, 592.

van Altena, W. F. and Auer, L. H. 1975, in Image Processing Techniques in Astronomy (edited by $C$. de Jager and $H$. Nieuwenhuizen), p. 411. Reidel, Dordrecht.

Wasserman, L. H., Bowell, E., and Millis, R. L. 1983, Astron. J. $\underline{88}$, 1670 .

\section{Discussion:}

EICHHORN:

occultation?

What is the uncertainty of the track for the Ceres

WASSERMAN:

The Ceres occultation will certainly be visible in Miami and probably will be visible here in Gainesville. The track is probably uncertain by less than $1 / 4$ of a track width. The star position was remeasured by Klemola; the asteroid ephemeris should be good. Final predictions will be possible in November when both objects will be on the same plate and can be measured with respect to a common set of stars. 